ISSN 0103-5150

Fisioter. Mov., Curitiba, v. 24, n. 1, p. 13-21, jan./mar. 2011 Licenciado sob uma Licença Creative Commons

\title{
Efeitos de um programa de reabilitação pulmonar sobre mecânica respiratória e qualidade de vida de mulheres obesas
}

\author{
Effects of a pulmonary rehabilitation program over respiratory \\ mechanics and life quality upon obese women
}

\section{Elisa Sonehara ${ }^{[a]}$, Maria do Socorro Luna Cruz ${ }^{[b]}$, Paula Roquetti Fernandes ${ }^{[c]}$, Fernando Policarpo ${ }^{[\mathrm{d}]}$, José Fernandes Filho ${ }^{[\mathrm{e}]}$}

[a] Fisioterapeuta, Mestre em Ciência da Motricidade Humana pela Universidade Castelo Branco (UCB-RJ), docente da Universidade Potiguar, Natal, RN - Brasil, e-mail: sonehara.elisa@hotmail.com

[b] Fisioterapeuta, Mestre em Ciência da Motricidade Humana pela Universidade Castelo Branco (UCB-RJ), docente da Universidade Potiguar, Natal, RN - Brasil.

[c] Doutora em Ciências da Saúde pela Universidade Federal do Rio Grande do Norte (UFRN), Centro de Excelência de Avaliação Física, Rio de Janeiro, RJ - Brasil.

[d] Doutor em Ciências da Saúde pela Universidade Federal do Rio Grande do Norte (UFRN), Centro de Excelência de Avaliação Física, Rio de Janeiro, RJ - Brasil; Universidade Trás-os-Montes e Alto Douro (UTAD) - Portugal.

[e] Doutor em Educação Física pelo Instituto de Pesquisa Científica de Cultura Física da Rússia, VNIIFK, docente da Universidade Federal do Rio de Janeiro (UFRJ), Rio de Janeiro, RJ - Brasil.

\section{Resumo}

Introdução: A obesidade é uma doença metabólica caracterizada por uma etiologia multifatorial que propicia o acúmulo de gordura levando a quadros de morbidade e mortalidade. Objetivo: Verificar os efeitos de um programa de reabilitação pulmonar sobre a mecânica respiratória, a capacidade funcional e a qualidade de vida de 20 mulheres obesas sedentárias com idade média de 48,75 $\pm 13,71$ e IMC de 34,54 $\pm 3,85 \mathrm{~kg} / \mathrm{m}^{2}$. Materiais e métodos: As voluntárias foram entrevistadas usando o questionário SF-36 como instrumento para determinar qualidade de vida, seguido da mensuração da massa corporal, estatura, cirtometria tóraco-abdominal, volume minuto e corrente, capacidade vital lenta, pressão inspiratória e expiratória máxima. A comparação dos resultados pré e pós-programa de reabilitação pulmonar foi pelo teste $t$ de Student pareado, observando-se um valor $\mathrm{p}<0,05$. Resultados: Os resultados após o programa foram estatisticamente significativos $(\mathrm{p}<0,05)$ para o volume minuto de $11008,30 \pm 5035,53 \mathrm{~mL}(\mathrm{p}=0,001)$, volume corrente de 758,70 $\pm 345,78 \mathrm{~mL}(\mathrm{p}=0,023)$, capacidade vital lenta com 2632,95 $\pm 852,25 \mathrm{~mL}(\mathrm{p}=$ 
0,001), pressão inspiratória máxima com $-69,75 \pm 21,37 \mathrm{cmH}_{2} \mathrm{O}(\mathrm{p}=0,045)$ e pressão expiratória máxima com 74,50 $\pm 28,23 \mathrm{cmH}_{2} \mathrm{O}(\mathrm{p}=0,000)$ e na percepção da qualidade de vida em seu estado geral de saúde $76,5 \pm 14,04(p=0,033)$, aspectos sociais 83,1 $\pm 18,71(p=0,031)$, aspectos emocionais 88,4 $\pm 19,52(p=$ 0,042 ) e na saúde mental $83,4 \pm 14,81(p=0,049)$. Conclusão: 0 programa de reabilitação pulmonar proposto promoveu efeitos positivos melhorando a mecânica respiratória e a qualidade de vida das mulheres obesas sedentárias.

Palavras-chave: Obesidade. Mecânica respiratória. Reabilitação pulmonar. Exercícios. Qualidade de vida.

\section{Abstract}

Introduction: Obesity is a metabolic disease that is characterized by a multifactorial etiology, which induces fat accumulation, oftentimes leading to morbid conditions or even death. Objective: The present study aims to verify the effects of a pulmonary rehabilitation program over respiratory mechanics, functional capacity, and life quality of 20 sedentary obese women aging in average $48.75 \pm 13.71$ e IMC de $34.54 \pm 3.85 \mathrm{~kg} / \mathrm{m}^{2}$. Materials and methods: The volunteers were submitted to an interview using the SF-36 questionnaire as a tool in order to determine the quality of life, take body mass measures, as well as stature, thoracic-abdominal cytometry, minute and volume tidal, slow vital capacity, and maximum and minimum inspiratory pressure. The comparison of results before and after the implementation of the pulmonary rehabilitation Program were tested by a paired Student's t-test, having been noted a $p<0.05$ value. Results: The results after the program were statistically significant $(p<0.05)$ for minute volume of $11008.30 \pm 5035.53 \mathrm{~mL}(p=0.001)$, volume tidal of $758.70 \pm 345.78 \mathrm{~mL}(p=0.023)$, slow vital capacity with $632.95 \pm 852.25 \mathrm{~mL}(p=0.001)$, maximum inspiratory pressures with $-69.75 \pm 21.37 \mathrm{cmH}_{2} \mathrm{O}(p=0.045)$ and maximum expiratory pressure with $74.50 \pm 28.23$ $\mathrm{CmH}_{2} \mathrm{O}(p=0.000)$, and in the perception of life quality concerning the general health condition $76.5 \pm 14.04$ ( $p=0.033)$, social aspects $83.1 \pm 18.71$ ( $p=0.031)$, emotional aspects $88.4 \pm 19.52(p=0.042)$, and in mental health $83.4 \pm 14.81$ ( $p=0.049$ ). Conclusion: The pulmonary rehabilitation program here proposed managed to promote positive effects, improving respiratory mechanics and life quality of sedentary obese women.

Keywords: Obesity. Respiratory mechanics. Pulmonary rehabilitation. Physical exercise. Life quality.

\section{Introdução}

A obesidade é a doença metabólica mais antiga que se tem conhecimento, é caracterizada pelo excesso de gordura corporal e sua etiologia multifatorial pode estar associada a distúrbios genéticos, endócrinos, hormonais, ambientais e socioeconômicos, bem como a hábitos alimentares desregrados e ao sedentarismo $(1,2)$. Sabe-se que a combinação desses fatores é determinante para o acometimento de doença crônica degenerativa não transmissível e que a manutenção do estilo de vida sedentário reduz a probabilidade de sucesso no tratamento da obesidade, sendo o sedentarismo responsável pelos altos índices de insucessos e recidivas, o que pode levar ao surgimento de transtornos psicossociais $(3,4)$.

O levantamento realizado pela Organização Mundial da Saúde (OMS) (5) aponta que mais de um bilhão de adultos estão em condições de risco graças ao excesso de peso e trezentos milhões apresentam quadro de obesidade, o que implica um problema de saúde pública. Esses resultados descrevem a importância da implantação de programas de prevenção primária e secundária que busquem diminuir o avanço dessa epidemia $(6,7)$.

A implantação de centros de reabilitação pode ser apontada como uma solução no tratamento de indivíduos com sobrepeso e obesidade, permitindo, assim, realizar as secundárias sobre as enfermidades comumente descritas na literatura (5). Além dos distúrbios sobre o sistema cardiovascular, o quadro de obesidade contribui, também, para alterações no fluxo ventilatório, ocasionando, em geral, distúrbios restritivos, o que em consonância a outros fatores determinantes favorece a morbidade e mortalidade $(5,8)$. As alterações ventilatórias acarretam redução do volume de reserva expiratório (VRE), diminuição da complacência pulmonar e torácica levando a 
quadros de dispneia, em função do acúmulo de gordura na região abdominal, provocando a compressão diafragmática e consequentemente dificultando a movimentação da caixa torácica na inspiração (9-12).

Tais alterações devem ser tratadas em programas específicos que procurem restabelecer os parâmetros funcionais como também promover mudanças significativas no estilo de vida. Dessa feita, a difusão de programas de reabilitação pulmonar é uma forma de contribuir para o gerenciamento adequado das enfermidades respiratórias nesse grupo de risco, tendo como base a formação de equipes multidisciplinares, com abordagens na reeducação alimentar, nos exercícios físicos e nas atividades fisioterapêuticas, o que possibilitará a recuperação do quadro de saúde (13).

Diante do exposto, este estudo teve por objetivo verificar os efeitos de um programa de reabilitação pulmonar sobre a mecânica respiratória, a capacidade funcional e a qualidade de vida de mulheres obesas sedentárias.

\section{Materiais e métodos}

0 presente estudo se caracteriza de forma descritiva, comparativa de abordagem longitudinal, em que se busca compreender as dimensões quantitativas e qualitativas que envolvem o processo de reabilitação pulmonar de indivíduos obesos $(14,15)$. Para tanto, foram selecionadas de forma intencional 20 voluntárias do sexo feminino, obesas e sedentárias, que estavam inscritas na lista de espera do Projeto Doce Vida da Universidade Potiguar (UnP), residentes na cidade de Natal, RN, com a participação sujeita aos seguintes critérios: ser voluntária; ter idade entre 18 e 70 anos; com IMC $\geq 30 \mathrm{~kg} / \mathrm{m}^{2}$; não ter doença pulmonar aguda ou crônica e apresentar estabilidade hemodinâmica mediante atestado médico, permitindo a participação no programa de reabilitação pulmonar; possuir capacidade de realizar as manobras e medidas fisioterapêuticas; e não estar em tratamento fisioterapêutico respiratório ou em programas de reabilitação semelhante. 0 projeto foi submetido e aprovado pelo Comitê de Ética em Pesquisa da Universidade Potiguar, RN, sob o registro no CEP/UnP n. 069/2006.

Após seleção, as voluntárias foram convidadas por telefone a comparecer na Clínica Escola do curso de Fisioterapia da Universidade Potiguar, Natal, RN, para uma reunião de esclarecimentos sobre o projeto, ocorrendo na sequência a leitura do termo de consentimento livre e esclarecido (TCLE), o qual todas assinaram. Em seguida, foram entrevistadas para preenchimento da ficha de identificação, antecedentes pessoais e familiares, medicações em uso. Para avaliar a percepção da qualidade de vida, foi utilizado o questionário Short-Form (SF-36), composto por 8 áreas de abrangência (16) fornecendo escore de $0 \mathrm{a}$ 100 , sendo 0 para pior estado de saúde e 100 representativo de ausência de problemas de saúde.

Em dia subsequente, as voluntárias foram submetidas à avaliação antropométrica da estatura e massa corporal, por meio de balança digital com estadiômetro modelo 2096 PP da marca Toledo ${ }^{\circledR}$ - Brasil, com limite de $150 \mathrm{~kg}$ e acuidade de $50 \mathrm{~g}$. As medidas foram realizadas com o mínimo de roupa possível e sem calçados. A partir desses valores foi calculado o índice de massa corpórea (IMC) em $\mathrm{kg} / \mathrm{m}^{2}$.

A determinação da cirtometria torácico-abdominal foi por trena da marca Sanny ${ }^{\circledR}$ Ind.- Brasil, com a voluntária na posição ortostática, tomando-se as circunferências nas região das axilas, do processo xifoide e da cicatriz umbilical, com solicitação de inspiração máxima ao nível da capacidade pulmonar total e expiração máxima até o volume residual (11).

A obtenção dos valores dos volumes corrente (VC) e minuto (VM) e da capacidade vital lenta (CVL) foi por meio do ventilômetro Ferraris Mark Wright Respirometer - USA, com limite operacional de 100 litros, acoplado a uma máscara facial de silicone da marca Vital Signs, Inc. - USA. Na medida do volume minuto (VM), a voluntária realizou inspirações e expirações normais e tranquilas por um período de um minuto. Durante esse tempo foi contabilizada a frequência respiratória (f) e as voluntárias encontravam-se sentadas para a mensuração desses parâmetros (17). 0 cálculo do volume corrente (VC) foi pela equação: $\mathrm{VC}=\mathrm{VM} / \mathrm{f}$, em que VM e f correspondem ao volume minuto e à frequência respiratória respectivamente. A mensuração da CVL foi realizada com a voluntária executando uma inspiração até a sua capacidade pulmonar total (CPT), seguida de uma expiração lenta sustentada pelo maior tempo possível, obtendo-se o valor em mililitros.

Para a determinação da pressão inspiratória e expiratória máxima, utilizou-se um manovacuômetro analógico da marca Gerar Ind. - Brasil, com limite operacional de $\left(-300 \mathrm{a}+300 \mathrm{cmH}_{2} \mathrm{O}\right)$, composto de 
um tubo flexível de PVC de $15 \mathrm{~cm}$ de comprimento e 1,5 mm de diâmetro acoplado a um bocal. No momento da mensuração, a voluntária estava sentada, com as narinas ocluídas por clipe nasal e, para a medida da pressão inspiratória máxima, foi solicitada uma expiração até o volume residual (VR), seguida de uma inspiração profunda até a capacidade pulmonar total, sustentada por três segundos (17), com o orifício do aparelho ocluído. A pressão expiratória máxima foi medida em $\mathrm{cmH}_{2} \mathrm{O}$, após uma inspiração profunda até a capacidade pulmonar total, seguida de uma expiração até o VR, com oclusão do orifício, sendo sustentada por três segundos (17). As medidas foram coletadas três vezes, com intervalo de descanso de 1 minuto entre as medidas, sendo computado o melhor resultado. 0 coeficiente de variação observado para a $\mathrm{PI}_{\max }$ e $\mathrm{PE}_{\max }$ é igual a $10,2 \%$ e $12,8 \%$ respectivamente (18).

Depois de concluída a mensuração das variáveis, iniciou-se o programa de reabilitação pulmonar de doze semanas, com frequência de três vezes semanais, com sessões de 60 minutos, o qual foi composto de aquecimento com alongamentos da musculatura cervical em flexo-extensão e inclinações laterais, sendo adotados os mesmos procedimentos para as cadeias musculares dos membros superiores, de tronco com inclinações laterais e, por último, cadeias musculares dos membros inferiores. Todos estes foram alongados em uma série de 30 segundos. 0 condicionamento aeróbico foi realizado em esteira ergométrica por 20 minutos, de maneira confortável, com intensidade de $70 \%$ da frequência cardíaca reserva (FCreserva) calculada pela fórmula de Karvonen et al. (1957): ([frequência cardíaca máxima - frequência cardíaca de repouso] $\times 70 \%+$ frequência cardíaca de repouso) (19).

O fortalecimento muscular dos membros superiores (MMSS) e cintura escapular foi realizado com auxílio de halteres de 2 e $3 \mathrm{~kg}$ para trabalhar as cadeias anterior, posterior, lateral e medial. Para fortalecer os músculos dos membros inferiores (MMII) foram realizados exercícios para todas as cadeias musculares com caneleiras de 1 e $2 \mathrm{~kg}$. A musculatura abdominal foi estimulada com exercícios em decúbito dorsal com pernas flexionadas seguido de flexão de tronco. Os exercícios foram realizados em três séries de quinze repetições com intervalos entre as séries de 60 segundos (20).

0 programa teve em sua estruturação sessões de relaxamento duas vezes por semana, com exercícios de alongamento globais consorciados a exercícios respiratórios: padrão diafragmático, soluços inspiratórios e apneia máxima pós-inspiratória. Uma vez por semana esse relaxamento foi realizado com treinamento da respiração diafragmática e conscientização do esquema corporal com aplicação de técnicas de relaxamento.

Ao término das doze semanas do programa de reabilitação, as voluntárias foram reavaliadas e seus resultados submetidos à análise estatística por meio do pacote estatístico SPSS versão 16 for Windows, em que a descrição dos resultados foi pela estatística de tendência central média e pelas medidas de dispersão dos dados desvio-padrão - DP (média \pm DP). Os dados obtidos durante o programa de reabilitação pulmonar foram submetidos ao teste de normalidade Kolmogorov-Smirnov. Com subsequente comparação dos resultados pré e pós-programa de reabilitação pulmonar pelo teste $t$ de Student pareado, sendo observado o nível de significância de $\mathrm{p}<0,05$.

\section{Resultados}

A amostra foi composta por 20 mulheres, obesas e sedentárias, com idade média de 48,8 $\pm 13,7$ anos, com a maior frequência de idade na faixa dos 50 aos 57 anos. As voluntárias não relataram, durante a entrevista, presença de doença pulmonar aguda ou crônica, porém em sua maioria tinham história de hipertensão arterial sistêmica controlada.

Os resultados obtidos neste estudo, correspondentes aos momentos pré e pós-programa, foram analisados em sua distribuição, estando dentro de uma curva de normalidade para um valor de $\mathrm{p}=0,05$.

As medidas para as variáveis massa corporal (MC), estatura e IMC estão descritas na Tabela 1. Apesar do programa de reabilitação pulmonar não ter como objetivo principal a redução da massa corporal, a análise das variáveis MC e IMC pós-programa indicou diminuição significativa $\mathrm{p}=0,03$ e $\mathrm{p}=0,02$, respectivamente, demonstrando que o programa teve influência no controle ponderal das voluntárias.

Os resultados para o exame físico apontaram que $80 \%$ (16) dos pacientes apresentaram um padrão respiratório costal e que $20 \%$ (4), costo-diafragmático. Após o programa de reabilitação pulmonar, 60\% (12) dos pacientes melhoraram seu padrão diafragmático, $30 \%$ (6) para o costo-dia- 
fragmático e $10 \%$ (2) dos pacientes permaneceram sem alterações.

A avaliação dos resultados da mecânica respiratória relacionada com a perimetria tóraco-abdominal apresentou diferença estatística significativa ( $\mathrm{p}<0,05)$ pós-programa para as variáveis: axilar e xifoide da expiração máxima. No entanto, as medidas axilar, xifoide e abdominal, durante a inspiração máxima e a medida abdominal na expiração máxima, não foram significativamente diferentes (Tabela 2).

Os resultados pós-programa de reabilitação para os volumes pulmonares minuto (VM), corrente (VC) e para a capacidade vital lenta (CVL) apontaram aumento significativo $(\mathrm{p}<0,05)$ quando comparados aos valores médios iniciais, sendo o ganho para o VM de 4.060,45 mL, o VC de 234,15 mL e a CVL de 906,05 mL (Tabela 3). Do mesmo modo, a força da musculatura respiratória representada pelas pressões inspiratória $\left(\mathrm{PI}_{\max }\right)$ e expiratória máxima $\left(\mathrm{PE}_{\max }\right)$ apresentou alterações significativas $(\mathrm{p}<0,05)$ de $-8,5 \mathrm{cmH}_{2} \mathrm{O}$ para $\mathrm{PI}_{\max }$ e de $+19,50$ $\mathrm{cmH}_{2} \mathrm{O}$ para $\mathrm{PE}_{\max }$, quando comparados os períodos pré e pós-programa de reabilitação (Tabela 3). Observaram-se ganhos tanto nas voluntárias que apresentavam volumes e capacidades respiratórias dentro da faixa de normalidade como para aquelas que estavam abaixo dos parâmetros normativos. No entanto, os resultados mais expressivos ocorreram para os valores mínimos, como pode ser analisado na Tabela 3, demonstrando a eficácia no método para o grupo amostral.

Com relação à qualidade de vida, os escores estabelecidos pelo SF-36 foram diferentes em todos os domínios do questionário da Tabela 4, porém, os domínios que apresentaram diferença significativa $(\mathrm{p}<0,05)$ foram: estado geral da saúde, aspectos sociais, aspectos emocionais e saúde mental, indicando alterações significativas no tocante à percepção da qualidade de vida pelas voluntárias.

Tabela 1 - Descrição dos valores da média, valor mínimo e máximo, de mulheres obesas sedentárias submetidas ao programa de reabilitação pulmonar

\begin{tabular}{lccccc}
\hline & & & & \multicolumn{2}{c}{ Média \pm DP } \\
\cline { 5 - 6 } Variáveis & $\mathbf{n}$ & Mín & Máx & Pré & Pós \\
\hline Idade (anos) & 20 & 18 & 68 & $48,75 \pm 13,71$ & $48,75 \pm 13,71$ \\
Massa corporal $(\mathrm{kg})$ & 20 & 66,0 & 115,7 & $83,34 \pm 12,10$ & $81,27 \pm 11,75^{\star}$ \\
Estatura $(\mathrm{m})$ & 20 & 1,44 & 1,66 & $1,55 \pm 0,05$ & $1,55 \pm 0,05$ \\
IMC $\left(\mathrm{kg} / \mathrm{m}^{2}\right)$ & 20 & 30,00 & 42,83 & $34,54 \pm 3,85$ & $33,72 \pm 3,87^{\star}$ \\
\hline
\end{tabular}

Legenda: $\mathrm{N}=$ amostra; $\mathrm{IMC}=$ índice de massa corpórea; * $=\mathrm{p}<0,05$.

Tabela 2 - Apresentação da média e do desvio-padrão (DP) para as variáveis da cirtometria tóracoabdominal de mulheres obesas no pré e pós-programa de reabilitação pulmonar (PRP)

\begin{tabular}{|c|c|c|c|c|}
\hline \multirow[b]{2}{*}{ Variáveis } & \multicolumn{2}{|c|}{ Média \pm DP } & \multirow{2}{*}{$\begin{array}{c}\text { Média } \pm \text { DP } \\
\text { Diferença pré-pós }\end{array}$} & \multirow[b]{2}{*}{ Sig. } \\
\hline & Pré & Pós & & \\
\hline Ax. insp (cm) & $106,00 \pm 8,92$ & $105,08 \pm 9,17$ & $0,93 \pm 2,62$ & 0,13 \\
\hline Xif. insp (cm) & $99,18 \pm 9,18$ & $98,02 \pm 9,01$ & $1,15 \pm 2,59$ & 0,06 \\
\hline Abd. insp (cm) & $104,08 \pm 9,48$ & $103,00 \pm 10,98$ & $1,08 \pm 4,24$ & 0,27 \\
\hline Ax. $\exp (\mathrm{cm})$ & $103,60 \pm 9,29$ & $102,02 \pm 9,71$ & $1,58 \pm 2,71$ & $0,02^{*}$ \\
\hline Xif. $\exp (\mathrm{cm})$ & $97,62 \pm 9,01$ & $95,95 \pm 8,65$ & $1,68 \pm 3,05$ & $0,02^{*}$ \\
\hline Abd. $\exp (\mathrm{cm})$ & $104,12 \pm 8,87$ & $102,90 \pm 9,79$ & $1,23 \pm 3,52$ & 0,14 \\
\hline
\end{tabular}

Legenda: $a x=$ axilar; xif = xifoide; abd = abdominal; insp = inspiração; $\exp =$ expiração; * $=p<0,05$. 
Tabela 3 - Apresentação da média, do desvio-padrão (DP) e do valor mínimo e máximo para as variáveis após a realização do programa de reabilitação pulmonar em mulheres obesas $(n=20)$ na cidade de Natal, RN

\begin{tabular}{lcrrcc}
\hline Variáveis & & Mín & Máx & Média \pm DP & Sig. \\
\hline VM $(\mathrm{mL})$ & Pré & 2390 & 22380 & $11008,30 \pm 5035,53$ & \\
& Pós & 6850 & 23258 & $15068,75 \pm 4365,98$ & $0,01^{*}$ \\
$\mathrm{VC}(\mathrm{mL})$ & Pré & 217 & 1570 & $758,70 \pm 345,78$ & \\
& Pós & 685 & 1595 & $992,85 \pm 308,53$ & $0,02^{*}$ \\
$\mathrm{CVL}(\mathrm{mL})$ & Pré & 900 & 4730 & $2632,95 \pm 852,25$ & \\
& Pós & 2200 & 4758 & $3539,45 \pm 728,57$ & $0,01^{*}$ \\
$\mathrm{PI}_{\text {max }}\left(\mathrm{CmH}_{2} \mathrm{O}\right)$ & Pré & -120 & -35 & $-69,75 \pm 21,37$ & \\
& Pós & -130 & -45 & $-78,25 \pm 21,42$ & $0,01^{*}$ \\
$\mathrm{PE}_{\text {max }}\left(\mathrm{CmH}_{2} \mathrm{O}\right)$ & Pré & 30 & 150 & $74,50 \pm 28,23$ & \\
& Pós & 40 & 150 & $94,00 \pm 24,74$ & $0,01^{*}$ \\
\hline
\end{tabular}

Legenda: $\mathrm{VM}=$ volume minuto; $\mathrm{VC}=$ volume corrente; $\mathrm{CVL}=$ capacidade vital lenta; $\mathrm{PI}_{\max }=$ pressão inspiratória máxima; $\mathrm{PE}_{\max }=$ pressão expiratória máxima; ${ }^{*}=\mathrm{p}<0,05$.

Tabela 4 - Valores obtidos no questionário de qualidade de vida SF-36 no pré e pósprograma de reabilitação pulmonar

\begin{tabular}{lccc}
\hline & \multicolumn{2}{c}{ Média \pm DP } & \\
\cline { 2 - 3 } Variáveis SF-36 & Antes & Depois & Sig. \\
\hline Capacidade funcional & $71,0 \pm 14,47$ & $73,3 \pm 21,72$ & 0,63 \\
Limitação por aspectos físicos & $70,0 \pm 36,81$ & $85,0 \pm 27,39$ & 0,17 \\
Dor & $63,0 \pm 20,79$ & $65,6 \pm 18,57$ & 0,64 \\
Estado geral de saúde & $69,3 \pm 17,05$ & $76,5 \pm 14,04$ & $0,03^{*}$ \\
Vitalidade & $71,3 \pm 16,53$ & $75,5 \pm 12,97$ & 0,27 \\
Aspectos sociais & $72,5 \pm 21,31$ & $83,1 \pm 18,71$ & $0,03^{*}$ \\
Aspectos emocionais & $68,4 \pm 35,07$ & $88,4 \pm 19,52$ & $0,04^{*}$ \\
Saúde mental & $78,2 \pm 13,07$ & $83,4 \pm 14,81$ & $0,04^{*}$ \\
\hline
\end{tabular}

Legenda: ${ }^{*}=p<0,05$.

\section{Discussão}

A mecânica respiratória ideal depende de um conjunto de estruturas agindo simultaneamente de maneira a garantir a ventilação, como: uma boa mobilidade torácica, força e propriedades dos músculos respiratórios íntegros e, consequentemente, bons volumes e capacidades pulmonares. No entanto, no obeso são encontradas alterações na mecânica respiratória, como a diminuição do volume de reserva expiratória e da capacidade residual funcional $(8,10)$, diminuição da complacência pulmonar e torácica, redução da força dos músculos respiratórios e distúrbios da função pulmonar e da capacidade de exercício (21), o que fica bem evidenciado no presente estudo pelos dados obtidos para o período pré-programa de reabilitação pulmonar.

Nos resultados observados neste estudo, a mudança do padrão respiratório costal para o diafragmático foi favorecida pela reabilitação pulmonar, 
que utilizou exercícios visando ao trabalho do músculo diafragma, buscando modificar o padrão respiratório anterior (costal) para o padrão fisiológico diafragmático. A mesma mudança respiratória também foi observada em um estudo realizado por Costa et al. (11) com 29 indivíduos obesos submetidos a uma avaliação da força muscular respiratória e de amplitudes torácicas após período de reeducação funcional respiratória. Após 18 sessões, observou-se mudança no padrão respiratório em decorrência, provavelmente, da adaptação da respiração diafragmática.

Os resultados obtidos no presente estudo demonstram que o programa de reabilitação pulmonar realizado no período de doze semanas teve eficácia em promover alterações significativas na mecânica respiratória de mulheres obesas, em função das alterações ocorridas na expansividade da cirtometria tóraco-abdominal, indicando melhora da mobilidade torácica e corroborando com o resultado de estudo predecessor (22), que foi aplicado em indivíduos com DPOC submetidos a um programa de exercícios físicos por dois meses - programa esse composto de exercícios respiratórios, com movimentos de tronco e fortalecimento dos músculos abdominais, que obtiveram aumento significativo na mobilidade na região inferior da caixa torácica.

Além disso, Powers e Howley (23) relataram em seus estudos que a prática de exercícios físicos, mesmo sendo de intensidade leve a moderada, pode exercer influência sobre as capacidades e perfusões pulmonares, justificando dessa forma as diferenças significativas $(p<0,05)$ encontradas neste estudo com o aumento de VC, VM, CVL, $\mathrm{PI}_{\max }$ e PE $\mathrm{P}_{\max }$ após a realização do programa de reabilitação pulmonar. Azeredo (24), em estudo que combinou os exercícios aeróbicos com alongamentos e exercícios ventilatórios diafragmáticos, obteve resultados que favoreceram o reequilíbrio da musculatura inspiratória e expiratória minimizando as alterações decorrentes da debilidade muscular, estimulando a atividade diafragmática e, assim, promovendo aumento do VC. Outro estudo relacionado com a prática de exercícios globais associados aos exercícios respiratórios dentro de um programa de reabilitação por meio da reeducação postural global, no período de 8 semanas, em jovens saudáveis e sedentários, ocasionou melhora significativa nas pressões respiratórias máximas e na mobilidade tóraco-abdominal (25).

A divergência observada para os dados obtidos no presente estudo foi para a $\mathrm{PE}_{\max }$ das obesas, que apresentou aumento significativo, opondo-se aos resultados descritos por Costa et al. (11), em que não houve diferenças significativas nos valores da $\mathrm{PE}_{\text {max }}$. No entanto, Costa et al. (11) citam que os valores médios obtidos da $\mathrm{PI}_{\max }$ apresentaram aumento significativo, o que é convergente com os resultados aqui apresentados, em que o aumento para $\mathrm{PI}_{\max }$ foi significativo. Essa melhora da $\mathrm{PE}_{\max }$ pode ser decorrente dos exercícios de fortalecimento da musculatura abdominal durante o programa de reabilitação, já que a $P E_{\max }$ é uma medida que indica a força dos músculos abdominais e intercostais (11).

Embora os resultados pertinentes à qualidade de vida não tenham mostrado melhora significativa ( $p<0,05)$ para os domínios capacidade funcional, aspectos físicos, vitalidade e dor, pode-se inferir que os escores obtidos apontam ganhos qualitativos, pois os escores médios apresentaram-se maiores após o programa de reabilitação pulmonar (PRP), mesmo estes não sendo estatisticamente significativos. Dessa forma, observa-se que o PRP melhorou a qualidade de vida das voluntárias. Esse resultado estabelece relação com os relatos de estudo anterior (26), em que foram entrevistados 69 universitários, de ambos os sexos, dos quais 33 praticavam musculação há três meses, e o grupo controle com 36 indivíduos pouco ativos. Ambos os grupos responderam ao questionário SF-36, objetivando relacionar a qualidade de vida com o bem-estar físico, social e emocional, por meio da prática de atividade física. Os resultados demonstraram que os domínios capacidade funcional, aspectos físicos, dor, vitalidade, estado geral da saúde, aspectos sociais e saúde mental apresentaram diferenças significativas no grupo feminino, o que é semelhante ao resultado observado no presente estudo. Esses resultados também podem ser decorrentes da realização das atividades físicas em grupo durante o programa. De acordo com Oliveira et al. (27), as orientações quando realizadas em grupo têm maior aproveitamento, pois, além do conhecimento teórico oferecido, também existe a troca de informações e vivência entre seus integrantes, favorecendo a ampliação nos relacionamentos (28). Além disso, Borges (29), em seu estudo realizado com idosos, observou que a prática de atividade física é de grande relevância na promoção de saúde, no aumento da autoestima e que, mesmo iniciada tardiamente, ainda oferece melhora da qualidade de vida e da capacidade funcional.

Sendo assim, o exercício físico pode melhorar e/ou minimizar as consequências da obesidade na 
capacidade funcional e no sistema cardiorrespiratório, principalmente em indivíduos com síndrome metabólica, melhorando a saúde e a qualidade de vida desses indivíduos (30). Embora os resultados tenham atingido os objetivos preestabelecidos, durante o decorrer do processo foram observadas algumas limitações pertinentes à execução, como a baixa aderência das voluntárias. A adequação das cargas de treinamento contrarresistido pode ser apontada como limitação, já que estas foram estimadas, o que reduz a capacidade de determinação da intensidade respeitando a individualidade biológica. Outros dois fatores limitantes do presente estudo foram a baixa acuidade observada para a cirtometria na aferição dos volumes pulmonares e, por fim, a ausência do grupo controle pertinente para melhor interpretação dos resultados obtidos no estudo. Como sugestão para estudos futuros, propõe-se a aplicação de protocolos que permitam estabelecer tanto a aptidão física neuromuscular quanto a determinação das cargas, como também possam ser utilizados como parâmetro de classificação. Seguindo o mesmo raciocínio, pode-se sugerir a avaliação da aptidão cardiorrespiratória por meio de método que permita maior acurácia na determinação desse parâmetro para um melhor planejamento das intensidades do treinamento aeróbico. Para tanto, sugere-se que as intensidades dos exercícios aeróbicos sejam estabelecidas tendo como parâmetro as respostas funcionais observadas em teste ergoespirométrico ou pela ventilometria de esforço.

Conclui-se, dessa forma, que a aplicação de programas de reabilitação pulmonar em mulheres obesas sedentárias favorece melhorias na mecânica respiratória e na qualidade de vida no nível de aptidão física, decorrentes das adaptações funcionais promovidas durante o período de 12 semanas, demonstrando que programas de reabilitação pulmonar aplicados em indivíduos que apresentam quadros de obesidade devem ser fomentados com o objetivo de prevenção secundária de doença degenerativa e promoção da saúde.

\section{Referências}

1. Bernardi F, Cichelero C, Vitolo MR. Comportamento de restrição alimentar e obesidade. Revista de Nutrição. 2005;18(1):85-93.
2. Sinisgalli LAM, Rodrigues HJ, Mair V. Cirurgia bariátrica. In: Sarmento GJ. Fisioterapia respiratória no paciente crítico: rotinas clínicas. São Paulo: Manole; 2005. p. 280-95.

3. Bernardi F, Cichelero C, Vitolo MR. Comportamento de restrição alimentar e obesidade. Revista de $\mathrm{Nu}$ trição. 2005;18(1):85-93.

4. Pinheiro ARO, Freitas SFT, Corso ACT. Uma abordagem epidemiológica da obesidade. Revista de Nutrição. 2004;17(4):523-33.

5. Organização Mundial de Saúde. Obesidade: prevenindo e controlando a epidemia global. São Paulo: Roca; 2003.

6. Negrão CE, Barretto ACP. Cardiologia do exercício: do atleta ao cardiopata. Barueri: Manole; 2005.

7. Marques APO, Arruda IKG, Espírito Santo ACG, Raposo MCF, Guerra MD, Sales TF. Prevalência de obesidade e fatores associados em mulheres idosas. Arq Bras Endocrinol Metab. 2005;49(3):441-8.

8. Koening SM. Pulmonary complications of obesity. Am J Méd Sci. 2001;321(4):249-79.

9. Stirbulov R. Repercussões respiratórias da obesidade. J Bras Pneumol. 2007;33(1):8-9.

10. Paisani DM, Chiavegato LD, Faresin SM. Volumes, capacidades e força muscular respiratória no pósoperatório de gastroplastia. J Bras Pneumol. 2005; 31(2):125-32.

11. Costa D, Sampaio LMM, Lorenzzo VAP, Jamami M, Damaso AR. Avaliação da força muscular respiratória e amplitudes torácicas e abdominais após a RFR em indivíduos obesos. Rev Latino-Am Enfermagem. 2003;1(2):156-60.

12. Rigatto AM, Alves SCC, Gonçalves CB, Firmo JF, Provin LM. Performance ventilatória na obesidade. Saúde Rev. 2005;7(17):57-62.

13. Organização Pan-Americana da Saúde - OPAS. Doenças crônico-degenerativas e obesidade: estratégia mundial sobre alimentação saudável, atividade física e saúde. Brasília: Organização Pan-Americana da Saúde; 2003.

14. Thomas Jr, Nelson JK. Métodos de pesquisa em atividade física. Porto Alegre: Artmed; 2002. 
15. Hochman B, Nahas FB, Oliveira Filho RS, Ferreira LM. Desenhos de pesquisa. Acta Cirúrgica Bras. 2005;20 (Supl 2):2-9.

16. Gonçalves FDP, Marinho PEM, Maciel MA, Galindo Filho VC, Dornelas de Andrade A. Avaliação da qualidade de vida pós-cirurgia cardíaca na fase I da reabilitação através do questionário MOS SF-36. Rev Bras Fisioter. 2006;10(1):121-6.

17. Sociedade Brasileira de Pneumologia e Tisiologia SBPT. Diretrizes para testes de função pulmonar. J Bras Pneumol. 2002;28(Supl 3):1-38.

18. Parreira VF, França DC, Zampa CC, Fonseca MM, Tomich GM, Britto RR. Pressões respiratórias máximas: valores encontrados e preditos em indivíduos saudáveis. Rev Bras Fisioter. 2007;11(5):361-8.

19. Policarpo FB, Cabral SAT, Montenegro Neto AN, Mayolino RB, Knackfuss MI, Fernandes PR, et al. Comparação de métodos para a determinação da intensidade do treinamento aeróbico para indivíduos jovens. Fit Perf J. 2007;6(6):367-70.

20. American College of Sports Medicine. American College of Sports Medicine position stand. Progression models in resistance training for healthy adults. Med Sci Sports Exerc. 2009;41(3):687-708.

21. Teixeira CA, Santos JE, Silva GA, Souza EST, Martinez JAB. Prevalência de dispnéia e possíveis mecanismos fisiopatológicos envolvidos em indivíduos com obesidade graus 2 e 3. J Bras Pneumol. 2007;33(1):28-35.

22. Paulin E, Brunetto AF, Carvalho CRF. Efeitos de programa de exercícios físicos direcionado ao aumento da mobilidade torácica em pacientes portadores de doença pulmonar obstrutiva crônica. J Pneumol. $2003 ; 29(5): 287-94$.

23. Powers KS, Howley TE. Fisiologia do exercício: teoria e aplicação ao condicionamento e ao desempenho. São Paulo: Manole; 2000.
24. Azeredo CA. Fisioterapia respiratória no Hospital Geral. São Paulo: Manole; 2000.

25. Moreno MA, Catai AM, Teodori RM, Borges BLA, Castro Cesar M, Silva E. Efeitos de um programa de alongamento muscular pelo método de reeducação postural global sobre a força muscular respiratória e a mobilidade toracoabdominal de homens jovens sedentários. J Bras Pneumol. 2007;33(6):679-86.

26. Macedo CSG, Garavello JJ, Oku EC, Miyagusuku FH, Agnoll PD, Nocett PM. Benefícios do exercício físico para a qualidade de vida. Rev Bras Ativ Fís Saúde. 2003;8(2):19-27.

27. Oliveira TC, Araújo TL, Melo EM, Almeida DT. Avaliação do processo adaptativo de um idoso portador de hipertensão arterial. Rev Latino-Am Enfermagem. 2002;10(4):530-6.

28. Chacra FC. Empatia e comunicação na relação médico-paciente: uma semiologia autopoiética do vínculo [tese]. Campinas: Faculdade de Ciência Médicas/ Unicamp; 2002.

29. Borges MRD, Moreira AK. Influências da prática de atividades físicas na terceira idade: estudo comparativo dos níveis de autonomia para o desempenho nas AVDs e AIVDs entre idosos ativos fisicamente e idosos sedentários. Motriz. 2009;15(3):562-73.

30. Santos JAR. Obesidade e exercício. Rev Bras Educ Fís Esp. 2006;20(Supl 5):1611-62.

Recebido: 08/04/2009

Received: 04/08/2009

Aprovado: $14 / 08 / 2010$

Approved: 08/14/2010 